\title{
Modellrechnungen über Platzwechselenergien in Kristalliten mit Zinkblendegitter
}

\author{
A. Bresch und F. Matossi \\ Institut für Elektrowerkstoffe der Fraunhofer-Gesellschaft, Freiburg i. Br. \\ und Institut für Physikalische Chemie der Universität Freiburg i. Br.
}

(Z. Naturforschg. 21 a, 465-469 [1966] ; eingegangen am 29. Januar 1966)

\begin{abstract}
With a simplified force system (Cобцомв and BoRn forces), the activation energies for several site exchanges in a small crystallite of cubic $\mathrm{ZnS}$ are computed. They depend strongly on the specific configuration of the crystallite.
\end{abstract}

Platzwechselvorgänge spielen in der Festkörperphysik eine große Rolle. Es ist deshalb von Interesse, im einzelnen die Aktivierungsenergien bei den Bewegungen von Ionen in einem Kristallgitter zu betrachten. Die Rechnungen, über die hier berichtet wird, wurden nicht für ein unendlich ausgedehntes Gitter durchgeführt, sondern es wurden sehr kleine Bereiche (mit 730 bzw. 56 Atomen oder Ionen) zugrunde gelegt. Das erschien gerechtfertigt, da an Aufdampfschichten bei tiefen Temperaturen im ersten Augenblick tatsächlich solche sehr kleinen Kristallite entstehen, wenn Atome aus der Dampfphase auf eine gekühlte Unterlage auftreffen ${ }^{1}$. Die Größe solcher Keime wurde zu etwa 125 Atomen abgeschätzt ${ }^{2}$.

Wir beschränken uns auf Platzwechsel von einem Gitterpunkt auf eine Leerstelle und von einem Gitterpunkt auf einen Zwischengitterplatz, der dadurch gekennzeichnet ist, daß die ungleichnamigen Ionen das herausgegriffene Ion in der gleichen Weise umgeben wie am Ursprung. Außerdem wurden Platzwechselvorgänge an der Kristallitoberfläche untersucht.

\section{Methode}

Bei der Bewegung eines Ions von einem Gitterplatz auf einen benachbarten Platz muß es sich im Inneren des Kristallgitters zwischen anderen Ionen hindurchbewegen, deren anziehende oder abstoßende Kräfte dann zur Wirkung kommen. Es muß also aus der Potentialmulde seiner Ausgangslage einen Potentialberg von der Höhe $A_{1}$ überwinden und fällt anschließend wieder in eine Potentialmulde, deren energetischer Abstand von der Ausgangs-

1 O. Fürst, R. Glocker u. H. Richter, Z. Naturforschg. 4 a, 540 [1949]. - K. Franck, Th. Müller u. K. Raithel, Optik 5,197 [1949]. lage $E$ betragen möge. Dazu braucht es eine Aktivierungsenergie $A_{1}$ in der einen Richtung und $A_{1}-E=A_{2}$ in der entgegengesetzten (Abb. 1). E bezeichnet man als freie Energie. Das Teilchen beschreibt dabei eine Kurve im Raum durch das Git-

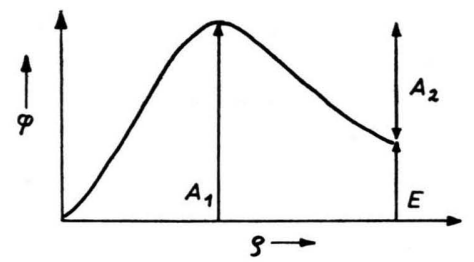

Abb. 1. Schema der Potentialkurve. $A_{1}, A_{2}=$ Aktivierungsenergien, $E=$ freie Energie.

ter. Der Schnitt des Potentialgebirges mit der Ebene dieser Kurve ergibt die für den Platzwechsel benötigte Aktivierungsenergie nur dann, wenn unter den an sich unendlich vielen Sprungmöglichkeiten der mit der kleinsten Aktivierungsenergie ausgewählt wird.

Das exakte mathematische Problem der Berechnung von Potentialkurven innerhalb und an der Oberfläche des Gitters besteht nun darin, die Energie $\varphi$ eines bestimmten Ions an seiner Ausgangslage $R_{0}$ zu bestimmen, das Ion um eine Strecke $\varrho$ in Richtung der zu berechnenden Raumkurve zu verschieben und dann an der Stelle $R^{\prime}=R_{0}+\varrho$ das Potential $\varphi^{\prime}$ des Teilchens zu berechnen. Dies Verfahren wird möglichst oft fortgesetzt bis zum nächsten Potentialminimum in der neuen Endlage des Ions. Die Bestimmung der Raumkurve des Teilchens mit der geringsten Aktivierungsenergie stellt natürlich ein kompliziertes Variationsproblem dar, besonders bei der großen Mannigfaltigkeit von Sprung-

2 T. Hoffmann, Festkörperphysik, Akademie-Verlag, Berlin 1961, S. 41 . 
möglichkeiten an der Oberfläche des Kristallits. Im allgemeinen dagegen (insbesondere im Inneren des Kristallits) sind die Platzwechsel geringster Aktivierungsenergie durch die Symmetrie des Gitters festgelegt und man kann oft durch qualitative Utberlegungen die richtige Raumkurve angeben.

Wir begnügen uns bei der Berechnung der Aktivierungsenergien mit СоuLoмв-Kräften und BoRNschen Abstoßungskräften. Außerdem vernachlässigen wir die Beiträge höherer Multipole. Für das Potential zwischen zwei Teilchen setzen wir deshalb folgende Gleichung an:

$$
\varphi=-e^{2} / r+b / r^{n}=\varphi_{\mathrm{C}}+\varphi_{\mathrm{A}} .
$$

In bekannter Weise ${ }^{3}$ ergibt sich für den unendlichen Kristall aus den Gleichgewichtsbedingungen für $b$ der Ausdruck

$$
b=M z^{2} e^{2} r_{0}{ }^{n-1} / n
$$

und mit Hilfe der Kompressibilität $\varkappa$ ergibt sich für kubische Kristalle ein Ausdruck von der Form

$$
n=1+\frac{9}{M z^{2} e^{2}} \cdot \frac{r_{0}^{4}}{\varkappa} .
$$

In diesen Gleichungen bedeuten $r_{0}$ den Gleichgewichtsabstand, $M$ die Madelung-Konstante, $z$ die Anzahl der Elementarladungen $e$ pro Ion, $b$ und $n$ Konstanten. Für das Zinkblendegitter ist dann $n \approx 5$. Führt man an Stelle von $b / r^{n}$ das BorN-Mayersche Potential $D e^{-r / \sigma}$ ein und setzt dies mit $\varphi_{\mathrm{A}}$ von (1) gleich, so erhält man mit $D=1,625 \cdot 10^{-9} \mathrm{erg}$ und $\sigma=0,370 \AA$ für $n$ den Wert $n=5,55$, mit dem wir als einer besseren Näherung weitergerechnet haben.

Bei der Berechnung von $\varphi$ für ein herausgegriffenes Teilchen als Funktion seiner Verschiebung $\varrho$ aus der Ausgangslage ist nun die Summe über die Beiträge aller berücksichtigten Ionen zu bilden. Dabei behalten wir die für den unendlichen Kristall erhaltenen Werte von $n$ und $b$ auch für den endlichen Kristallit bei, was zweifellos nicht exakt gültig sein muß, aber als plausible Näherung erlaubt sein dürfte.

Die hier beschriebenen Näherungen und Vernachlässigungen lassen natürlich keine exakten Werte für die Absolutwerte der Aktivierungsenergien erwarten. Trotzdem wird dieses vereinfachte Modell qualitative Vergleiche verschiedener Aktivierungsenergien erlauben.

3 G. Leibfried, Handbuch der Physik VII/1, herausgeg. von S. FLÜGGE, Springer-Verlag, Berlin 1955, S. 130.

\section{Ergebnisse}

Zunächst wurde ein Würfel des $\mathrm{ZnS}$ als Gitter mit 64. Elementarzellen und insgesamt $730 \mathrm{Zn}^{++}$. und $\mathrm{S}^{--}$-Ionen gewählt. Der Würfel ist so aufgebaut, daß abwechselnd $\mathrm{Zn}$ - und S-Schichen aufeinander folgen. Gegenüberliegende Würfel-Oberflächen sind dann mit entgegengesetzt geladenen Ionen besetzt. Infolge dieser Asymmetrie nimmt das Potential im ganzen von einer Seite zur anderen ab. Der Einfluß der Oberfläche auf ein Ion im Inneren ist also keineswegs vernachlässigbar. $\mathrm{Da}$ aber überhaupt diese Anordnung wenig wahrscheinlich erscheint, wurde eine möglichst symmetrische Konfiguration mit insgesamt 56 Ionen der weiteren Rechnung zugrunde gelegt.

Bei einem Modell von 56 Ionen verändert aber schon die Versetzung eines einzigen Ions unter Umständen grundlegend die Potentialkurve. Wir wählten eine Konfiguration, bei der die Ionen möglichst symmetrisch um die Punkte 11 und 12 angeordnet sind (Abb. 2). Die kartesischen Koordinaten dieser Ausgangskonfiguration sind aus den Tabellen 1 und 2 ersichtlich.

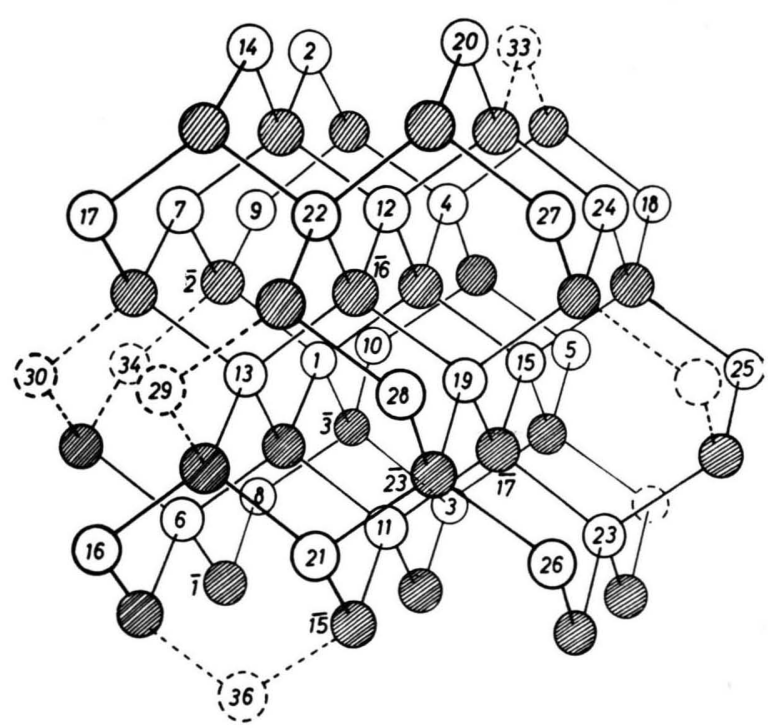

Abb. 2. Kristallit-Modell (Ausgangskonfiguration). Zn: $\bigcirc$, S: Kreis mit Schraffierung. Die gestrichelt gezeichneten Plätze sind unbesetzt. Die Ziffern beziehen sich auf im Text oder in den Tabellen erwähnte Punkte. Aus Gründen der Ubersichtlichkeit sind jedoch nicht alle im Text erwähnten Leerstellen in die Abbildung aufgenommen worden. Die Punkte I, $\overline{2}$ und I5 definieren das Koordinatensystem. 


\begin{tabular}{|r|r|r|r|r|r|r|r|}
\hline 1 & $1 / 4$ & $1 / 4$ & $3 / 4$ & 15 & $3 / 4$ & $3 / 4$ & $3 / 4$ \\
2 & $1 / 4$ & $1 / 4$ & $7 / 4$ & 16 & $3 / 4$ & $-3 / 4$ & $1 / 4$ \\
3 & $1 / 4$ & $3 / 4$ & $1 / 4$ & 17 & $3 / 4$ & $-3 / 4$ & $5 / 4$ \\
4 & $1 / 4$ & $3 / 4$ & $5 / 4$ & 18 & $3 / 4$ & $5 / 4$ & $5 / 4$ \\
5 & $1 / 4$ & $5 / 4$ & $3 / 4$ & 19 & $5 / 4$ & $1 / 4$ & $3 / 4$ \\
6 & $1 / 4$ & $-1 / 4$ & $1 / 4$ & 20 & $5 / 4$ & $1 / 4$ & $7 / 4$ \\
7 & $1 / 4$ & $-1 / 4$ & $5 / 4$ & 21 & $5 / 4$ & $-1 / 4$ & $1 / 4$ \\
8 & $-1 / 4$ & $1 / 4$ & $1 / 4$ & 22 & $5 / 4$ & $-1 / 4$ & $5 / 4$ \\
9 & $-1 / 4$ & $1 / 4$ & $5 / 4$ & 23 & $5 / 4$ & $3 / 4$ & $1 / 4$ \\
10 & $-1 / 4$ & $3 / 4$ & $3 / 4$ & 24 & $5 / 4$ & $3 / 4$ & $5 / 4$ \\
11 & $3 / 4$ & $1 / 4$ & $1 / 4$ & 25 & $5 / 4$ & $5 / 4$ & $3 / 4$ \\
12 & $3 / 4$ & $1 / 4$ & $5 / 4$ & 26 & $7 / 4$ & $1 / 4$ & $1 / 4$ \\
13 & $3 / 4$ & $-1 / 4$ & $3 / 4$ & 27 & $7 / 4$ & $1 / 4$ & $5 / 4$ \\
14 & $3 / 4$ & $-1 / 4$ & $7 / 4$ & 28 & $7 / 4$ & $-1 / 4$ & $3 / 4$ \\
\hline
\end{tabular}

Tab. 1. Kartesische Koordinaten der $\mathrm{Zn}^{++}$-Ionen.

\begin{tabular}{|c|c|c|c||c|c|c|c|}
\hline$\overline{1}$ & 0 & 0 & 0 & $\overline{15}$ & 1 & 0 & 0 \\
$\overline{2}$ & 0 & 0 & 1 & $\overline{16}$ & 1 & 0 & 1 \\
$\overline{3}$ & 0 & $1 / 2$ & $1 / 2$ & $\overline{17}$ & 1 & $1 / 2$ & $1 / 2$ \\
$\overline{4}$ & 0 & $1 / 2$ & $3 / 2$ & $\overline{18}$ & 1 & $1 / 2$ & $3 / 2$ \\
$\overline{5}$ & 0 & $-1 / 2$ & $1 / 2$ & $\overline{19}$ & 1 & $-1 / 2$ & $1 / 2$ \\
$\overline{6}$ & 0 & 1 & 1 & $\overline{20}$ & 1 & $-1 / 2$ & $3 / 2$ \\
$\overline{7}$ & $1 / 2$ & 0 & $1 / 2$ & $\overline{21}$ & 1 & 1 & 0 \\
$\overline{8}$ & $1 / 2$ & 0 & $3 / 2$ & $\overline{22}$ & 1 & 1 & 1 \\
$\overline{9}$ & $1 / 2$ & $1 / 2$ & 0 & $\overline{23}$ & $3 / 2$ & 0 & $1 / 2$ \\
$\overline{10}$ & $1 / 2$ & $1 / 2$ & 1 & $\overline{24}$ & $3 / 2$ & 0 & $3 / 2$ \\
$\overline{11}$ & $1 / 2$ & $-1 / 2$ & 0 & $\overline{25}$ & $3 / 2$ & $1 / 2$ & 0 \\
$\overline{12}$ & $1 / 2$ & $-1 / 2$ & 1 & $\overline{26}$ & $3 / 2$ & $1 / 2$ & 1 \\
$\overline{13}$ & $1 / 2$ & 1 & $1 / 2$ & $\overline{27}$ & $3 / 2$ & $-1 / 2$ & 1 \\
$\overline{14}$ & $1 / 2$ & 1 & $3 / 2$ & $\overline{28}$ & $3 / 2$ & 1 & $1 / 2$ \\
\hline
\end{tabular}

Tab. 2. Kartesische Koordinaten der $\mathrm{S}^{--}$-Ionen.

\section{A. Oberflächenplatzwechsel}

Zunächst befaßten wir uns mit Platzwechseln an der Oberfläche wegen der damit zusammenhängenden Fragen der Oberflächendiffusion, die vor allem beim Zusammenwachsen sehr kleiner Bereiche zu Mikrokristalliten bei der Sublimation aus der Dampfphase durch Einschwingvorgänge eine wichtige Rolle spielen. Es wurden verschiedene Platzwechsel Gitter-Zwischengitter sowie Gitter - Leerstellen für $\mathrm{Zn}^{++}$-Ionen bestimmt. Zwischen Ausgangsund Endlage wurden jeweils an 3 dazwischenliegenden, äquidistanten Punkten das Potential berechnet. Dabei wurden folgende Leerstellen bzw. Zwischengitterplätze berücksichtigt:

$$
\begin{array}{lll}
\text { Leerstelle } & 29: & 5 / 4,-3 / 4,3 / 4 ; \\
\text { Leerstelle } & 30: & 1 / 4,-3 / 4,3 / 4 ; \\
\text { Zwischengitterplatz } & 31: & 5 / 4,-3 / 4,5 / 4 ; \\
\text { Zwischengitterplatz } & 32: & 3 / 4,-3 / 4,3 / 4
\end{array}
$$

Die betrachteten Plätze sind durch Abb. 2 und 3 näher gekennzeichnet.

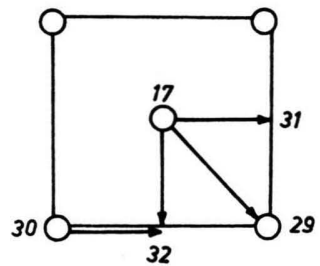

Abb. 3. Oberflächen-Platzwechsel.

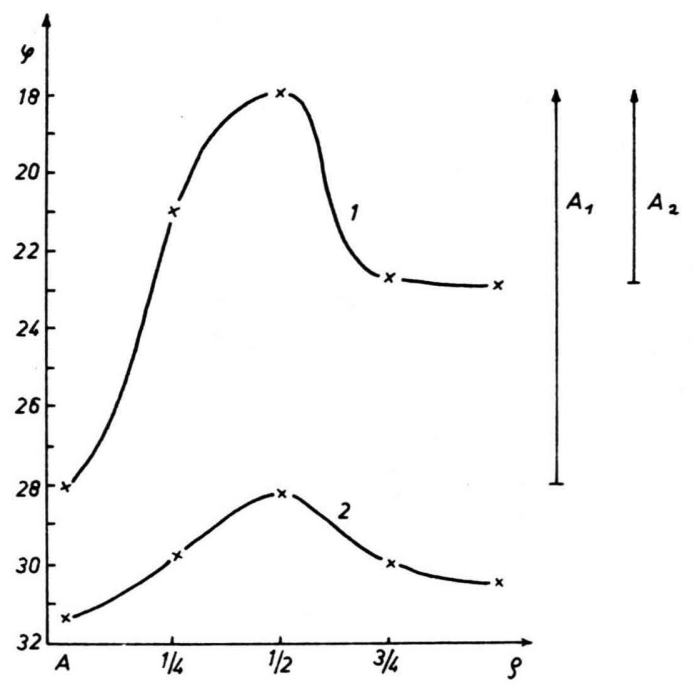

Abb. 4. Potentialkurven zu Tab. 3. $\varrho=$ Entfernung des Aufpunkts von der Ausgangslage in Bruchteilen der Gesamtverschiebung. 1 und 2 entsprechen der Bezeichnung in Tab. 3.

a) Platzwechseltyp: Gitter - Leerstelle, $17 \rightarrow 29$.

Es wurden 2 Fälle durchgerechnet:

1. mit der Ausgangskonfiguration (Tab. 1 u. 2),

2. Gitterpunkt 16 zur Leerstelle $33(3 / 4,3 / 4,7 / 4)$ verschoben; sonst wie 1 .

Das Ergebnis zeigt Tab. 3 und Abb. 4.

\begin{tabular}{|c|rc|}
\hline & 1 & 2 \\
\hline$A_{1}(\mathrm{eV})$ & 10,04 & 3,26 \\
$A_{2}(\mathrm{eV})$ & 5,10 & 2,19 \\
$E(\mathrm{eV})$ & 4,94 & 1,07 \\
\hline
\end{tabular}

Tab. 3. Platzwechsel Gitterpunkt-Leerstelle $(17 \rightarrow 29)$.

b) Platzwechseltyp: Gitter - Zwischengitter.

Für diese Art von Platzwechsel wurden im ganzen 6 Fälle mit verschiedenen Konfigurationen behandelt:

1. $17 \rightarrow 31$ mit Ausgangskonfiguration,

2. $17 \rightarrow 32$, 2 Fälle mit verschiedenen Konfigurationen,

3. $30 \rightarrow 32$, 2 Fälle mit verschiedenen Konfigurationen; 
zu 2.:

a) Ausgangskonfiguration,

$\beta)$ Gitterpunkt 14 zur Leerstelle $34(-1 / 4$, $-1 / 4,3 / 4$ ) verschoben, sonst wie $\alpha$ ),

$\gamma$ ) Konfiguration wie $\beta$ ), aber Gitterpunkt 16 nun nach Gitterplatz 14 verschoben;

zu 3.:

a) vor dem Platzwechsel $30 \rightarrow 32$ wurde Gitterpunkt 17 nach 30 versetzt; sonst wie Ausgangskonfiguration,

$\beta$ ) Konfiguration wie $\alpha$ ), aber Gitterpunkt 14 nach Leerstelle 34 verschoben.

Es zeigt sich (Tab. 4), daß je nach Konfiguration ein Zwischengitterplatz sogar energetisch günstiger liegen kann als ein Gitterplatz. In diesem Fall war übrigens der höchste Punkt der Kurve deutlich zum Ausgangspunkt des Platzwechsels hin verschoben.

\begin{tabular}{|l|rrrrrr|}
\hline & 1 & $2 \alpha$ & $2 \beta$ & $2 \gamma$ & $3 \alpha$ & $3 \beta$ \\
\hline$A_{1}(\mathrm{eV})$ & 6,17 & 4,62 & 8,83 & 5,05 & 3,80 & 4,21 \\
$A_{2}(\mathrm{eV})$ & 1,02 & 1,80 & 0,83 & 9,65 & 0,96 & 2,26 \\
$E(\mathrm{eV})$ & 5,15 & 2,82 & 8,00 & $-4,61$ & 2,84 & 1,95 \\
\hline
\end{tabular}

Tab. 4. Platzwechsel Gitterpunkt-Zwischengitter an der Oberfläche.

\section{B. „Innere“ Platzwechsel}

Neben den reinen Oberflächenplatzwechseln betrachteten wir auch Platzwechsel, die mehr im „Inneren" des Kristallbereichs liegen, soweit dieser Begriff hier noch gerechtfertigt ist. Es handelt sich dabei vor allem um Platzwechsel, die in Richtung der Achse durch die Ionen 11 und 12 und senkrecht dazu erfolgen (Abb. 5). Bei diesen Platzwechseln wurde

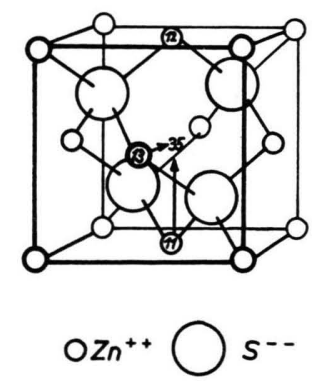

Abb. 5. Platzwechsel im Kristallit-Inneren, $35=$ Zwischengitterplatz.

aus Symmetriegründen jeweils nur 1 Punkt in der Mitte der Verschiebungslinie berechnet. Es wurden verschiedene Fälle sowohl mit unterschiedlicher $\mathrm{Zn}^{++}$. Ionen als auch $\mathrm{S}^{--}$-Ionenkonfiguration behandelt. a) Platzwechseltyp: Gitterpunkt $11 \rightarrow$ Zwischengitterpunkt $35(3 / 4,1 / 4,3 / 4)$ parallel zur Achse $11-12$.

Für diesen Platzwechsel wurden insgesamt 9 Fälle mit verschiedenen Konfigurationen berechnet (Tab. 5) :

1. mit Ausgangskonfiguration,

2. $\mathrm{Zn}^{++}$-Gitterpunkt 14 versetzt nach Leerstelle $36(3 / 4,-1 / 4,-1 / 4)$; sonst wie 1 .,

3. $\mathrm{Zn}^{++}$-Gitterpunkt 12 versetzt nach Leerstelle $37(7 / 4,-3 / 4,5 / 4)$; sonst wie 1 . (damit wird in nächster Nähe des Zwischengitterplatzes eine Leerstelle erzeugt),

4. $\mathrm{S}^{--}$-Gitterpunkt $\overline{2} \overline{3}$ versetzt nach Leerstelle $\overline{2} \overline{9}(1,-1 / 2,-1 / 2)$, sonst wie 3 .

5. $\mathrm{S}^{--}$-Gitterpunkt $\overline{3}$ versetzt nach Leerstelle $\overline{3} \overline{0}(3 / 2,1 / 2,2)$; sonst wie 4 .,

6. $\mathrm{S}^{--}$-Gitterpunkt $\overline{3}$ versetzt nach Leerstelle $\overline{3} \overline{1}(1,3 / 2,-1 / 2)$; sonst wie 5 .,

7. $\mathrm{S}^{--}$-Gitterpunkt $\mathrm{I} \overline{7}$ nach Gitterpunkt $\overline{3}$; sonst wie 6.

8. $\mathrm{S}^{--}$-Gitterpunkt $\bar{I} \overline{7}$ versetzt nach Leerstelle $\overline{3} \overline{2}(1,-1,1)$; sonst wie 5 .,

9. $\mathrm{S}^{--}$-Gitterpunkt $\overline{1} \overline{7}$ versetzt nach Leerstelle $\overline{3} \overline{2}$; sonst wie 3 .

\begin{tabular}{|l|ccccccccc|}
\hline & 1 & 2 & 3 & 4 & 5 & 6 & 7 & 8 & 9 \\
\hline$A_{1}(\mathrm{eV})$ & \multicolumn{1}{|c}{19,25} & 12,43 & 5,42 & 6,29 & 4,74 & 4,68 & 5,82 & 5,91 & 6,09 \\
$A_{2}(\mathrm{eV})$ & 0 & 0 & 1,55 & 2,79 & 2,24 & 2,62 & 1,22 & 3,30 & 2,01 \\
$E(\mathrm{eV})$ & & & 3,87 & 3,50 & 2,50 & 2,06 & 4,60 & 2,61 & 4,08 \\
\hline
\end{tabular}

Tab. 5. Platzwechsel $11 \rightarrow 35$.

b) Platzwechsel $13 \rightarrow 35$, senkrecht zur Achse $11-12$.

Für diesen Platzwechsel wurden insgesamt 6 verschiedene Fälle berechnet:

1. mit Ausgangskonfiguration,

2. $\mathrm{Zn}^{++}$-Gitterpunkt 20 versetzt nach Leerstelle 29;

$\mathrm{Zn}^{++}$-Gitterpunkt 2 versetzt nach Leerstelle 30;

3. $\mathrm{Zn}^{++}$-Gitterpunkt 15 nach Gitterpunkt 20 versetzt; sonst wie 2 .,

4. $\mathrm{Sn}^{--}$-Gitterpunkt $\overline{1} \bar{\sigma}$ versetzt nach Leerstelle $\overline{3} \overline{4}(1 / 2,-1 / 2,2)$; sonst wie 2 .,

5. $\mathrm{S}^{--}$-Gitterpunkt $\overline{1} \overline{6}$ versetzt nach Leerstelle $\overline{3} \overline{4}$; sonst wie 3 .

Hier ergeben sich nur Energiestufen, ohne dazwischenliegende Potentialberge (Tab. 6).

\begin{tabular}{|c|ccccc|}
\hline & 1 & 2 & 3 & 4 & 5 \\
\hline$A_{1}(\mathrm{eV})$ & 6,43 & 5,45 & $-3,20$ & 5,86 & $-2,55$ \\
\hline
\end{tabular}

Tab. 6. Platzwechsel $13 \rightarrow 35$. 
Bei den Fällen 3. und 5. ist der Ausgangsgitterplatz instabil und die Zwischengitterplätze liegen günstiger; daher das negative Vorzeichen für die Aktivierungsenergie.

\section{Platzwechsel Zwischengitter - Zwischengitter mit zusätzlichem $\mathrm{Zn}^{++}$-Ion}

Es wurden 2 Fälle durchgerechnet, bei denen die Stöchiometrie dadurch verletzt wurde, daß ein Ion zuviel in den Kristallit eingebaut wurde. Dies läßt sich immer erreichen, solange es unbesetzte Zwischengitterplätze gibt (Tab. 7).

a) Platzwechsel $35 \rightarrow 38(5 / 4,-1 / 4,3 / 4)$, wobei auf Platz 35 das zusätzlich hinzugefügte $\mathrm{Zn}^{++}$. Ion sitzt;

b) Platzwechsel $35 \rightarrow 39(5 / 4,1 / 4,(3 / 4)$.

Platz 38 entspricht der rechten vorderen Kantenmitte der Abb. 5, Platz 39 der rechten oberen Kantenmitte.

\begin{tabular}{|c|cc|}
\hline & $\mathrm{a}$ & $\mathrm{b}$ \\
\hline$A_{1}(\mathrm{eV})$ & 6,60 & 4,82 \\
$A_{2}(\mathrm{eV})$ & 5,06 & 1,08 \\
$E(\mathrm{eV})$ & 1,54 & 3,74 \\
\hline
\end{tabular}

Tab. 7. Platzwechsel Zwischengitter - Zwischengitter.

Im unendlichen Gitter wären diese beiden Platzwechsel einander gleichwertig; im endlichen Kristallit sind sie es nicht.

\section{Diskussion}

Die Aktivierungsenergien der untersuchten Platzwechsel zeigen eine starke Abhängigkeit von der Konfiguration, was wohl auch zu erwarten war. Bei den Oberflächenplatzwechseln fallen die berechneten Aktivierungsenergien im Durchschnitt etwas niedriger aus (sie liegen im großen und ganzen in der Größenordnung von $4-6 \mathrm{eV} / \mathrm{Mol}$ ) als im „Inneren“ des Bereichs, wo sie Werte um $6 \mathrm{eV} / \mathrm{Mol}$ zeigen. Der Unterschied ist aber viel geringer als man vielleicht

4 S. Asano u. Y. Tomishima, J. Phys. Soc. Japan 13, 1119 [1958]. erwartet hätte, und besonders überrascht der hohe Wert von $A_{1}$ in Tab. 3 , wobei man aber bedenken muß, daß der Punkt 29 von 17 aus auch auf anderen Wegen erreicht werden kann, z. B. über 32 (Tab. 4, Fall 2). Unsere Ergebnisse lassen sich gut mit denjenigen von Asano und Tomishima ${ }^{4}$ in Einklang bringen. Diese Autoren berechnen unter Hinzunahme von kovalenten Kräften für den unendlich ausgedehnten Kristall Aktivierungsenergien von 4,5 bis $6 \mathrm{eV}$. Sie gehen dabei vom Energieminimum der Gesamtkonfiguration aus. Bei unseren Beispielen gingen wir dagegen von willkürlich angenommenen Konfigurationen aus, wie sie im ersten Augenblick der Kondensation aus der Dampfphase bei sehr kleinen Kristalliten vorhanden sein dürften. Im allgemeinen entsprechen diese Konfigurationen nicht dem Energieminimum von Atomen, die den Bereich zusammensetzen. Wie die Tab. $3-6$ zeigen, gibt es Aktivierungsenergien, die niedrig genug sind, daß auch bei relativ tiefen Temperaturen Platzwechselvorgänge stattfinden können, die zu einer energetisch günstigeren Konfiguration führen. Sogar negative "Aktivierungsenergien“ kamen vor. Bei den „inneren" Platzwechseln sind jedoch einige Werte um das 2- bis 3-fache größer (Tab. 5). Dies wird durch die besonders große Ordnung in der Nähe des herausgegriffenen Ions bestimmt. Bei stärker gestörter Umgebung fallen die Aktivierungsenergien wie die freien Energien wesentlich niedriger aus. Man darf daraus schließen, daß Störungen im Kristallaufbau durch Besetzung von Zwischengitterplätzen schon im Stadium der ersten Kristallkeimbildung angelegt werden, während in einigermaßen geordneter Umgebung neue Störungen dieser Art praktisch nicht mehr entstehen werden.

Im Vergleich zu diesen Rechnungen ergaben einige vor kurzer Zeit durchgeführte Messungen ${ }^{5}$ an $\mathrm{A}^{\mathrm{III}} \mathrm{B}^{\mathrm{V}}$. und $\mathrm{A}^{\mathrm{II}} \mathrm{B}^{\mathrm{VI}}$-Verbindungen für $\mathrm{Zn}^{++}$-Ionen im $\mathrm{ZnSe}$ 9,9 eV/Mol und für $\mathrm{Cd}^{++}$-Ionen im CdS 7,3 eV/Mol als Aktivierungsenergien. Dabei handelt es sich aber um größere Kristallbereiche.

5 R. Bäuerlein, Z. Phys. 176, 498 [1963]. 